\title{
Improved Hybrid Model in Vehicular Clouds based on Data Types (IHVCDT)
}

\author{
Saleh A. Khawatreh \\ Dept. of Computer Engineering \\ Faculty of Engineering, Amman University \\ Amman-Jordan
}

\author{
Enas N. Al-Zubi \\ Dept. of Computer Engineering \\ Faculty of Engineering, Amman University \\ Amman, Jordan
}

\begin{abstract}
In Vehicular Cloud (VC), vehicles collect data from the surrounding environment and exchange this data among the vehicles and the cloud centers. To do that in an efficient way first we need to organize the vehicles into clusters, each one works as a VC, and every cluster is managed by the cluster head (broker). The vehicles are grouped in clusters with adaptive size based on their mobility and capabilities. This model is an approach that forms the clusters based on the vehicles capabilities and handles with different types of data according to its importance to select the best route. A hybrid model is proposed to deal with these differences; Long-Term Evolution (LTE) is used with IEEE 802.11P which forms the traditional wireless access for Vehicular Ad hoc Networks (VANETs). This merge gives the high data delivery, wide-range transmission, and low latency. However, using only LTE based VANET is not practical due to its high cost and the large number of occurrences in the base stations. In this paper, a new Vehicular Cloud (VC) model is proposed which provides data as a service based on Vehicular Cloud Computing (VCC). A new method is proposed for high data dissemination based on the data types. The model is classified into three modes: the urgent mode, the bulk mode, and the normal mode. In the urgent mode, Long-Term Evolution (LTE) is used to achieve a high delivery with minimum delay. In the bulk mode, the vehicle uses IEEE 802.11p and chooses two clusters to divide this huge data. In the normal mode, the model works as D-hops cluster based algorithm.
\end{abstract}

Keywords-Vehicular Cloud (VC); Vehicular Cloud Computing (VCC); Vehicular Ad hoc Networks (VANETs); cloud algorithms; hybrid transmissions; IEEE 802.11p; Long-Term Evolution (LTE); transmission cost

\section{INTRODUCTION}

In recent years, Vehicular Cloud Computing (VCC) has attracted the concern of researchers to deploy the Intelligent Transportation System (ITS). VCC is the concept of merging Mobile Cloud Computing (MCC) and Vehicular Ad-hoc Networks (VANETs). MCC is the study of the characteristics of mobile agents (people, vehicles, robots). The mobile agents interact and collaborate among each other to sense the surrounding environment, process the data, propagate and aggregate it. The result of the interaction is to be shared among the network where this cannot be done using conventional Internet Cloud. On the other hand, VANET is a type of networks which consists of networks of vehicles to achieve a specific purpose.
VANETs have been established as an efficient network in which vehicles communicate among each other on highways and urban environments.

Nowadays, most vehicles are equipped with embedded systems, integrated computers, processing units and sensors. All these improvements provide a good platform to deliver Data as a Service (Daas), therefore, providing an efficient and timely data diffusion about such events as traffic jams, accidents, and road conditions. In VANETs, the mobility of vehicles, different network density and the frequent changes in topologies are the most challenges that must be considered. In addition to these challenges, real time applications are strict to delay and data delivery of these safety messages. Up to now, most VANETs have applied IEEE 802.11p as a communication method, which forms the conventional way for wireless access. IEEE $802.11 \mathrm{p}$ supplies data rate range from 6 to $27 \mathrm{Mb} / \mathrm{s}$ at a short transmission distance, $300 \mathrm{~m}$. Diffusion safety messages over a huge area needs an intelligent multi-hop broadcast techniques dealing with broadcasting overhead and frequent disconnections.

Recently, cellular technology has been used as an alternative to IEEE $802.11 \mathrm{p}$. Due to the standardization of advanced broadcast/multicast in the Third-Generation (3G) which is called a Universal Mobile Communication System (UMCS), provides efficient and dynamic data dissemination over the network. As the rapid improvements on the communications, the Fourth Generation (4G) which is called Long-Term Evolution (LTE) is presented to support high data rates up to $300 \mathrm{Mb} / \mathrm{s}$ for downlinks, and up to $75 \mathrm{Mb} / \mathrm{s}$ for uplinks, with low delay of less than $5 \mathrm{~ms}$, for up to $100 \mathrm{Km}$ transmission range. Although these high benefits, LTE is not used alone in VANETs due to the high cost of communications between the vehicles among each other and between them and the base stations, take in mind the mobility of the vehicles and the high overload on the base station.

In recent years, hybrid solutions have been presented to achieve the benefits of the two merging techniques; IEEE $802.11 \mathrm{p}$ and LTE. Therefore, we obtain the low cost, the high data rate, high transmission range, and low delay [16].

In this paper, a new improved model is presented compared with the DHCV [15] which is based on the use of the vehicles capabilities and the hybrid transmission for data dissemination. This model is the first one that takes vehicles capabilities into consideration when clustering the vehicles. In this model, the 
clustering technique is used to organize the exchange of data between vehicles and between the vehicles and the base stations. Every cluster is formed based on the QoS of the data collected from the sensors, and selects the $\mathrm{CH}$ based on its mobility and capabilities. After the clusters are performed, the Cluster Member (CM) which has an emergency data uses the LTE to transmit this data; therefore, we insure the delivery of this important data with low latency.

Section II presents most of the related works. Section III describes the system model. Section IV provides comparison scenarios and evaluation of the simulation results. Finally, Section $\mathrm{V}$ presents some concluding remarks and provides future work.

\section{RELATED WORKS}

Several studies have been proposed in VANET in general, and in data allocation routing specifically. In this section, a review of some existing methods is presented. To have a better understanding of these methods two categories of cluster-based algorithms have been studied so far; the first one is based on location; where speed, location, and direction of movement are used for cluster formation. The second one is based on computable collective parameters, for example, network density and connectivity. The most existing cluster-based algorithms concerning with these categories form one-hop cluster. Such as, Stability Based Clustering Algorithm (SBCA) [1] where this algorithm is one-hop cluster and constructing clusters based on the relative mobility among the vehicles and the accessibility of the Cluster Head $(\mathrm{CH})$. To obtain this stability a Secondary Cluster Head ( $\mathrm{SCH})$ is selected along with the cluster head, this algorithm chooses Carrier Sense Multiple Access with Collision Avoidance (CSMA/CA) for transmissions, therefore a frequent contention occurs.

CSMA/CA is the basic for the MAC protocol which operates in IEEE 802.11p. MAC protocol is not the efficient way to use due to the features of VANETs such as vehicles mobility interference and hidden nodes. In situations of high vehicle density, the vehicles wait for a long time for sharing medium access, therefore, the network will suffer from low data throughput and long delay. Many protocols were presented to address these problems, such as; Space Division Multiple Access |(SDMA) protocol [2] which allocates different frequency ranges to the space units in the cluster. AdHoc MAC protocol [3] makes the transmission scheduling according to assign time slots to the vehicles which aim to access the medium. So the performance of these protocols decreases in high dense networks due to the hidden node and the congestion. Many protocols presented to address these problems, one of them is Clustering-based multichannel MAC protocol [4], this protocol is one-hop clusters approach that makes some improvements on the MAC layer by clustering the vehicles into clusters and let the cluster head control the cluster member's transmission in the shared medium, and support real time data transmission and not-real time data within quality of service. Therefore, the congestion is decreased compared with the previous protocols.

Hidden node problem is solved by Cluster-based MAC protocol (D-CBM) [5] that clusters the vehicles according to their mobility; it uses CSMA/CA or TDMA to schedule the transmission. In [6], a transmission based on QoS-TDMA is proposed; this protocol assigns pre-reserved slots of time that satisfy the priority. In [7] TDMA cluster-based MAC protocol assigns different slots to the cluster members in its one hop, therefore a fairness is achieved. These MAC protocols address the intra-cluster transmissions without collision.

All the above algorithms are one-hop clusters. These clusters have small coverage range; therefore, the movement of vehicles will reconstruct the clusters. On the other hand, multihop clusters achieve better performance due to its stability and the decrement of reconstructions.

Hierarchical Clustering model (HC) [8] is a randomized clustering algorithm. It clusters the vehicles based on the connectivity data among the neighbors without using a GPS to locate their locations. The size of the clusters is limited to two hops only. $\mathrm{HC}$ algorithm constructs the clusters in the first stage and does the adjustments in the maintenance phase. HC algorithm does not consider the mobility of the vehicles and that was the major drawback of it. In [9] a Modified Distributed Mobility-Adaptive Cluster (Modified DMAC) is proposed. It improves DMAC [10] by clustering the vehicles which only have the same direction of movement. The major drawback here is it does not consider the mobility of the vehicles in selecting the cluster heads. In [11], a Distributed Multi-Hop Clustering Scheme for VANETs based on a neighborhood Follow (DMCNF). In this algorithm the vehicles follow their one-hop neighbors according to these factors: the historical cluster membership, relative mobility, and the number of followers. The clusters in DMCNF tend to be large and this decreases the network throughput and increases the delay due to the large number of cluster members and that makes a bottleneck in the cluster heads. In [12] a multi-hop clustering model is proposed. It clusters the vehicles based on the relative mobility between them in multi-hop range. Each node (vehicle) selects its cluster head in at most D hops. Beacon messages are exchanged among the vehicles within the $\mathrm{D}$ hops, and each node calculates the beacon delay. The node with the least delay among D hops broadcasts itself as a cluster head. This algorithm improves the stability of the clusters by avoid reconstructions of the clusters when two cluster heads are located within D hops. The high overhead is the major drawback of this algorithm. In [13], a Vehicular Multi-Hop Algorithm for Stable Clustering (VMSC) is proposed. In this algorithm, the least mobility vehicle is selected as a cluster head to provide the clustering stability. To do that vehicles calculate the average speed of the vehicles within D hops. The drawback here is the high overhead as in the previous one. In [14] a Vehicular Deterministic medium Access controls (VDA). It schedules transmissions up to two hops, therefore, decreases the transmission delay and the collisions. In [15], a D-Hops Clustering Vehicles (DHCV) is proposed. In this algorithm the vehicles are grouped into D hops clusters according to location and speed differences between the vehicles within its D range. Each vehicle has a GPS to obtain its speed and location and to broadcast these data by WAVE standard to its neighbors within D hops. After the cloud construction, the cluster head manages the transmission scheduling inside the cluster by mathematical optimization model. DHCV provides better performance than the previous 
algorithms, due to, its stability and the usage of both physical layer and Medium Access Control (MAC) layer to schedule transmissions.

The main drawbacks of DHCV, it cannot work with real time applications and urgent data, due to its cluster-based model, and as known, one of the characteristic of VANET is the frequent re-clustering due to the mobility of vehicles. Another case, when the vehicle has a huge data, the model does not achieve a high throughput with low delay. There are other drawbacks that DHCV did not conceder such as, the availability of the $\mathrm{CH}$ and the vehicle capabilities.

Finally, in this paper some improvements are proposed to DHCV, first include the vehicle capability into consideration when electing the cluster head. Second, I propose a merging technique of IEEE802.11p and Long-Term Evolution (LTE) to transmit real time data. Third, let the vehicles which have a lot of data be $\mathrm{CM}$ in two clusters to ensure its delivery and to collaborate among the nodes.

\section{SYSTEM MODEL}

An Improved Hybrid model in Vehicular Clouds based on Data Types (IHVCDT) is the unique model which checks the data type and according to this data it will decide which mode to operate.

\section{A. System Modes}

As shown in Fig. 1, we have three different modes which classified according to the type of data.



Fig. 1. The system modes.

\section{1) The Urgent Mode}

This mode operates if the data is urgent such as, huge accident, earthquake, and road crashes. The most important metric to be concerned here is the delay so we must deliver the data with the minimum delay. To obtain this goal, the vehicle that has an urgent data will assume itself as a $\mathrm{CH}$ or a separate vehicle and transmit this data directly to the nearest base station using LTE.
2) The Bulk Mode

If the data is huge such as long video, and is not urgent to be sent, the vehicle chooses a $\mathrm{CH}$ that has an efficient capabilities including:

- Availability: It means whether this $\mathrm{CH}$ has the ability to accept this CM or not.

- Bandwidth: It is the data rate which is based on the following factors: interfaces of the devices, transmission medium, the weather, and the service provided by the Internet Service Provider (ISP).

- Vehicle capabilities: It is the vehicle can handle allot of requests, the size of the buffer it has, the cost of transmissions.

In this case, the most optimization here is the cost. The vehicles choose two $\mathrm{CHs}$ according to the previous factors. After cloud creation, the vehicle will be a CM in two clusters, and divide the data between them. The transmission of data to the $\mathrm{CH}$ is done by IEEE 802.11p. The equation to select the $\mathrm{CH}$ according to transmission cost is as follows:

Let Cxy, denotes the cost difference between $\mathrm{X}$ and $\mathrm{Y}$.

$$
\mathrm{Cxy}=|\mathrm{Cx}-\mathrm{Cy}| \quad \text { where } \mathrm{y} \in \mathrm{N}(\mathrm{x})
$$

Where $\mathrm{Cx}$ and $\mathrm{Cy}$ present the transmission cost of nodes $\mathrm{X}$ and Y.

\section{3) The Normal (Routine) Mode}

This mode operates when the data is small and not urgent such as advertisements, fuel stations locations, hotels, etc.

This mode operates as an optimized DHCV [15], where the clusters are created based on the distance differences between the vehicles and after the cloud creation a mathematical optimization managed by the $\mathrm{CH}$ controls the transmissions from the $\mathrm{CM}$ to $\mathrm{CH}$ and from $\mathrm{CH}$ to base stations.

\section{PERFORMANCE EVALUATIONS}

In this section, the expected performance evolutions are presented. The expectation results obtained from this research are compared with these simulation results that obtained in DHCV [15]. The simulator was NS2 to evaluate the proposed DHCV. The transcendence choice of the vehicles is considered as the velocity limit, the distance between the vehicle in front of it, and acceleration of the vehicles.

The authors of DHCV [15] compared the optimization scheduling technique between CSMA/CA and VDA [14]. Based on throughput and delay, VDA is a deterministic model to access the medium that schedules the transmission in contention free durations up to two hops. Two VANETs are used, one is low density which has 2 vehicles per $\mathrm{km}$, the other is high density with 12 vehicles per $\mathrm{km}$. Here, all the vehicles have the same transmitting power, which is $5 \mathrm{~mW}$. The other parameters are listed below in Table 1 . 
TABLE I. SIMULATION PARAMETERS

\begin{tabular}{|l|l|}
\hline Parameters & Value \\
\hline Propagation model & Nakagami \\
\hline System bandwidth & $10 \mathrm{MHz}$ \\
\hline Message payload size & $500 \mathrm{byte}$ \\
\hline MAC and PHY & $802.11 \mathrm{p}$ \\
\hline Noise power density & $-131 \mathrm{dbm}$ \\
\hline Raw bitrate & 1 to $6 \mathrm{Mbps}$ \\
\hline Modulation & BPSK $1 / 2$ \\
\hline Simulation time & $10 \mathrm{sec}$ \\
\hline Vehicle speed & $40 \mathrm{~km} / \mathrm{h}$ \\
\hline
\end{tabular}

To evaluate the performance, the following metrics are considered:

1) The average data throughput: is the average data that is received from the $\mathrm{CMs}$ to the $\mathrm{CHs}$.

2) The average delay: is the average time needed from sending the message from $\mathrm{CMs}$ to $\mathrm{CHs}$.

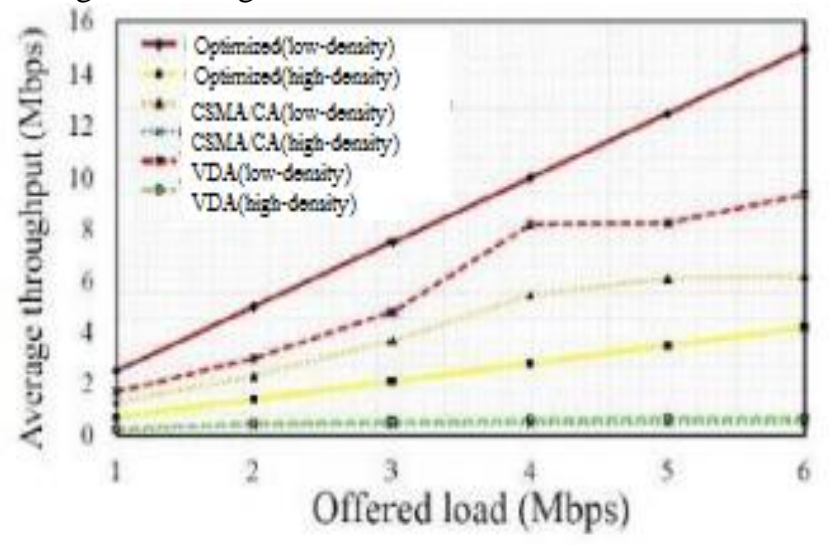

Fig. 2. Average throughput.

\section{A. Throughput}

Fig. 2 presents the average throughput with the different loads. As shown in Fig. 2, as the load increases the throughput increases. Due to the fact that as the load increases the data delivery will also increase. According to the results, the optimized DHCV has the best delivery in all scenarios due to many facts: the transmission links are optimally scheduled to decrease the contentions, the $\mathrm{CH}$ is responsible about the transmissions inside the cloud, and the hidden node is addressed in the optimized DHCD, therefore, it obtains the best result among the compared models [15].

\section{B. End to End Delay}

Fig. 3 presents the average end-to-end delay with different loads. As shown in Fig. 3, as the load increases the delay deceases. According to these results, the optimized DHCV model has the lowest delay in all scenarios due to the fact that the optimized DHCV solves the hidden nodes and interference problems, by $\mathrm{CH}$ which considers the physical condition of the medium [15].

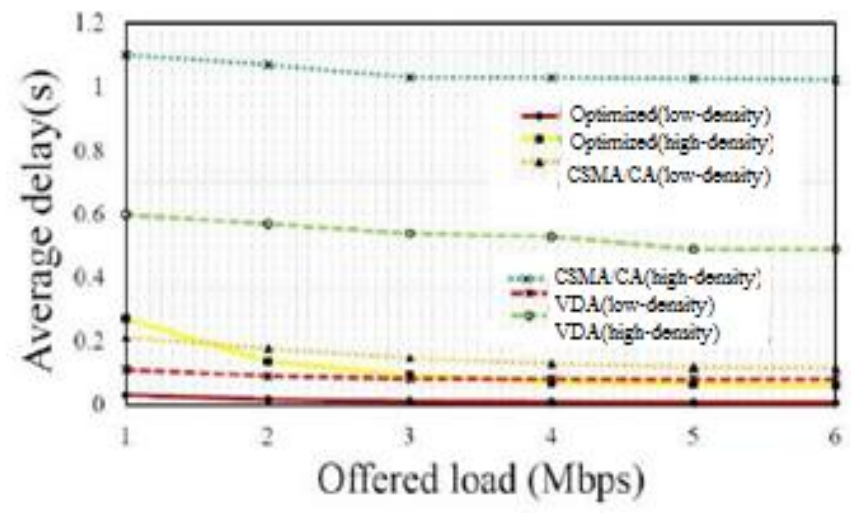

Fig. 3. Average end to end delay.

If the simulation results [15] are compared with IHVCDT, best performance is obtained in the urgent case and in the bulk case, where in the normal case the same results of the optimized DHCV will be obtained. These perspectives are considered from the advantage of using LTE instead of IEEE $802.11 \mathrm{p}$ in the first case, and of the participation of the vehicle into two clusters as in the second case. While, in the third case, the vehicles operate as in the optimized DHCV [15], and according to their results, it has the best throughput. And the minimum delay ever. As a result, if the normal case has the best results then our improved model will be the best of all.

\section{CONCLUSION}

The model presented in this paper is an Improved Hybrid model in Vehicular Clouds based on Data Types (IHVCDT). This model expected to provide the best delivery ratio within the minimum latency. The model is based on the data classification. The model is classified into three cases; the first one, is the urgent mode where data is urgent, and therefore, the concern here is to obtain a high data delivery with low latency. The vehicle which has an urgent data assumes itself as a cluster head or a separate vehicle and sends the data directly to the cloud station (base station) by using LTE. The second case, is the bulk mode, where the data is huge where the vehicle chooses the most efficient $\mathrm{CH}$ which has an efficient capability such as; the processor units in its interface, bandwidth, availability, and its transmission cost. All these factors should be considered to select the $\mathrm{CH}$. In this case, the vehicle can be participated in two clusters to divide this huge data between the clusters to insure its delivery. The transmission here is based on IEEE 802.11p. The third case is the normal (routine) mode, where the data is small and not urgent. Here, the vehicle chooses the $\mathrm{CH}$ based on the distance as in DHCV [15]. This mixture produces a new hybrid model that provides the advantages of LTE and IEEE 802.11p.

The main drawback of this model is that each vehicle should have two interfaces for transmission, one for LTE, and one for IEEE $802.11 \mathrm{p}$.

As future work, as the mobility in VANETs can be predictable, we propose to make the $\mathrm{CH}$ selection is based on the vehicles mobility in addition to the proposed factors, this provides another QoS model, where vehicles can send a prerequisite to a specific $\mathrm{CH}$ to become its $\mathrm{CH}$. 


\section{REFERENCES}

[1] Ahizoune, Ahmed, and Abdelhakim Hafid. "A new stability based clustering algorithm (SBCA) for VANETs." Local Computer Networks Workshops (LCN Workshops), 2012 IEEE 37th Conference on. IEEE, 2012.

[2] Bana, Soheila V., and Pravin Varaiya. "Space division multiple access (SDMA) for robust ad hoc vehicle communication networks." Intelligent Transportation Systems, 2001. Proceedings. 2001 IEEE. IEEE, 2001.

[3] Borgonovo, Flaminio, et al. "ADHOC MAC: new MAC architecture for ad hoc networks providing efficient and reliable point-to-point and broadcast services." Wireless Networks 10.4 (2004): 359-366.

[4] Su, Hang, and $\mathrm{Xi}$ Zhang. "Clustering-based multichannel MAC protocols for QoS provisionings over vehicular ad hoc networks." IEEE Transactions on Vehicular Technology 56.6 (2007): 3309-3323.

[5] Mammu, Aboobeker Sidhik Koyamparambil, Unai Hernandez-Jayo, and Nekane Sainz. "Cluster-based MAC in VANETs for safety applications." Advances in Computing, Communications and Informatics (ICACCI), 2013 International Conference on. IEEE, 2013.

[6] Mu'azu, Abubakar Aminu, et al. "A QoS approach for cluster-based routing in VANETS using TDMA scheme." 2013 international conference on ICT convergence (ICTC). IEEE, 2013.

[7] Almalag, Mohammad S., Stephan Olariu, and Michele C. Weigle. "Tdma cluster-based mac for vanets (tc-mac)." World of Wireless, Mobile and Multimedia Networks (WoWMoM), 2012 IEEE International Symposium on a. IEEE, 2012.

[8] Dror, Efi, Chen Avin, and Zvi Lotker. "Fast randomized algorithm for hierarchical clustering in vehicular ad-hoc networks." Ad Hoc Networking Workshop (Med-Hoc-Net), 2011 The 10th IFIP Annual Mediterranean. IEEE, 2011.
[9] Wolny, Grzegorz. "Modified DMAC clustering algorithm for VANETs." 2008 Third International Conference on Systems and Networks Communications. IEEE, 2008.

[10] Basagni, Stefano. "Distributed clustering for ad hoc networks." Parallel Architectures, Algorithms, and Networks, 1999.(I-SPAN'99) Proceedings. Fourth InternationalSymposium on. IEEE, 1999.

[11] Chen, Yuzhong, et al. "Distributed multi-hop clustering algorithm for VANETs based on neighborhood follow." EURASIP Journal on Wireless Communications and Networking 2015.1 (2015): 1-12.

[12] Zhang, Zhenxia, Azzedine Boukerche, and Richard Pazzi. "A novel multi-hop clustering scheme for vehicular ad-hoc networks." Proceedings of the 9th ACM international symposium on Mobility management and wireless access. ACM, 2011.

[13] Ucar, Seyhan, Sinem Coleri Ergen, and Oznur Ozkasap. "VMaSC: Vehicular multi-hop algorithm for stable clustering in vehicular ad hoc networks." 2013 IEEE Wireless Communications and Networking Conference (WCNC). IEEE, 2013.

[14] Rezgui, Jihene, Soumaya Cherkaoui, and Omar Chakroun. "Deterministic access for dsrc/802.11 p vehicular safety communication." 2011 7th International Wireless Communications and Mobile Computing Conference. IEEE, 2011.

[15] Azizian, Meysam, Soumaya Cherkaoui, and Abdelhakim Hafid. "An Optimized Flow Allocation in Vehicular Cloud." IEEE Access 4 (2016): 6766-6779.

[16] Ucar, Seyhan, Sinem Coleri Ergen, and Oznur Ozkasap. "MultihopCluster-Based IEEE 802.11 p and LTE Hybrid Architecture for VANET Safety Message Dissemination." IEEE Transactions on Vehicular Technology 65.4 (2016): 2621-2636. 